\title{
The X-ray side of the absorption by interstellar dust in the Milky Way
}

\section{E. Costantini*}

SRON, Netherlands Institute for Space Research, Sorbonnelaan, 2, 3584CA, Utrecht, The Netherlands

E-mail: e.costantiniesron.nl

\section{C.P. de Vries}

SRON, Netherlands Institute for Space Research, Sorbonnelaan, 2, 3584CA, Utrecht, The Netherlands

E-mail: c.p.de.vriesesron.nl

\section{S.T. Zeegers}

SRON, Netherlands Institute for Space Research, Sorbonnelaan, 2, 3584CA, Utrecht, The Netherlands

E-mail: s.t.zeegersesron.nl

\section{F. de Groot}

University Utrecht, Debye Institut Nanomaterial Science, Inorganic Chemistry and Catalysis Group, NL-3584 CG Utrecht, Netherlands

E-mail: f.m.f.degrootauu.nl

\section{H. Mutschke}

Laboratory Astrophysics Group of the Astrophysical Institute and University Observatory, Friedrich Schiller University Jena Schillergässchen 3, D-07743 Jena, Germany

E-mail: harald.mutschkeduni-jena.de

\section{Pinto}

IoA, Madingley Road, Cambridge, CB3 OHA, United Kingdom

E-mail: cpintodast.cam.ac.uk

\section{R. Waters}

SRON, Netherlands Institute for Space Research, Sorbonnelaan, 2, 3584CA, Utrecht, The Netherlands

E-mail: 1.b.f.m.watersesron.nl 
X-ray spectroscopy of interstellar dust (ID), seen in absorption against spectra of bright background sources, is a powerful tool to investigate and provide novel information on the chemistry of dust grains. For example, sharp and deep absorption features of $\mathrm{Mg}, \mathrm{Si}, \mathrm{O}$ and $\mathrm{Fe}$, which are the building blocks of silicates, fall in the X-ray band. Therefore X-ray spectroscopy can also successfully study the nature of the iron inclusion in dust. The shift and the structure of a given absorption feature is indeed different for different absorbing dust grains. High-quality data have already revealed that $\mathrm{Mg}$-rich silicates are favored with respect to the Fe-rich population and that $\mathrm{Fe}$ is most likely in metallic form. The modeling of spectra of present and future X-ray facilities will take now advantage of our new laboratory measurements, from several species, of the absorption features which are key for a complete understanding of the ID chemistry.

The Life Cycle of Dust in the Universe: Observations, Theory, and Laboratory Experiments - LCDU 2013, 18-22 November 2013

Taipei, Taiwan

${ }^{*}$ Speaker. 


\section{Introduction}

Interstellar dust (ID) takes part in every important process in the Universe, from our own solar system formation to star formation in galaxies at cosmological distance. Dust plays a fundamental role in the star life-cycle. It is indeed present in the primordial cloud which eventually collapses in a stellar body. Later on, dust is newly created within outflowing winds from giant stars and within the catastrophic supernovae explosions. Decades of observations from radio to the far-ultraviolet allowed us to understand the fundamental characteristics of the interstellar dust in our Galaxy [5]. However, some fundamental issues remain open, like for instance the ratio between the production and destruction rates of dust [6], the oxygen depletion [8] and the iron inclusion in dust [11,7].

The study of interstellar dust in the X-ray band may contribute to solve some of these issues. In the X-ray band, for instance, the absorption edges of the important constitutents of the silicates, including iron, are available for direct spectroscopy. X-rays are also sensitive to a large range of column densities $\left(N_{\mathrm{H}} \sim 10^{20-23} \mathrm{~cm}^{-2}\right.$ ), allowing us to explore different dust environments. Finally, all our Galaxy can be investigated, as the strong X-ray sources, which are used as background light to illuminate the intervening dust, are naturally distributed all over the Galactic plane.

\section{Recent results}

The launch of the ESA mission XMM-Newton and the NASA mission Chandra, marked the beginning of new investigations in X-ray astronomy in general, and in the study of absorption and scattering by interstellar dust in particular. For example, dust scattering halos of diffuse emission, could be studied with unprecedented spacial resolution $[2,18,15,16]$. Thanks to the high-energy resolution instruments, absorption could be studied in greater detail. The effect of absorption by dust is to shift and modify the shape of the photoelectric edges (Fig.2). This is due to the interaction of the incoming photoelectrons wave and the atoms inside the grain, creating extra absorption near the edge (called X-ray absorption fine structure). From the shape and position of those structures, the compound responsible for the absorption can be in principle derived [10, 3, 14, 17].

The current study of ID in the X-rays, directly investigating the deep absorption edges of oxygen and iron, is now delivering a very different scenario on the dust chemistry of the diffuse interstellar dust than what was believed up to now. Along different lines of sight in the Galactic plane there is striking evidence of Mg-rich silicates rather than Fe-rich [2, 10,3], against what was commonly assumed [11]. In [3], a simultaneous study of the $\mathrm{O} \mathrm{K}$ and Fe L absorption edges offered for the first time the evidence that that Fe can take in fact the form of metallic iron, possibly included in Mg-silicates (as proposed in [1]). Interestingly, this would be in agreement with the condensation history of the elements around late-type stars [7].

Higher column density environments may not have the same chemical composition. An abundance gradient across the Galaxy has been measured for the main dust constituents [12] and therefore lines of sights crossing regions near the Galactic Center may display an overabundance of e.g. iron with respect to the diffuse medium. Preliminary results on the Si edge analysis of the X-ray spectrum 


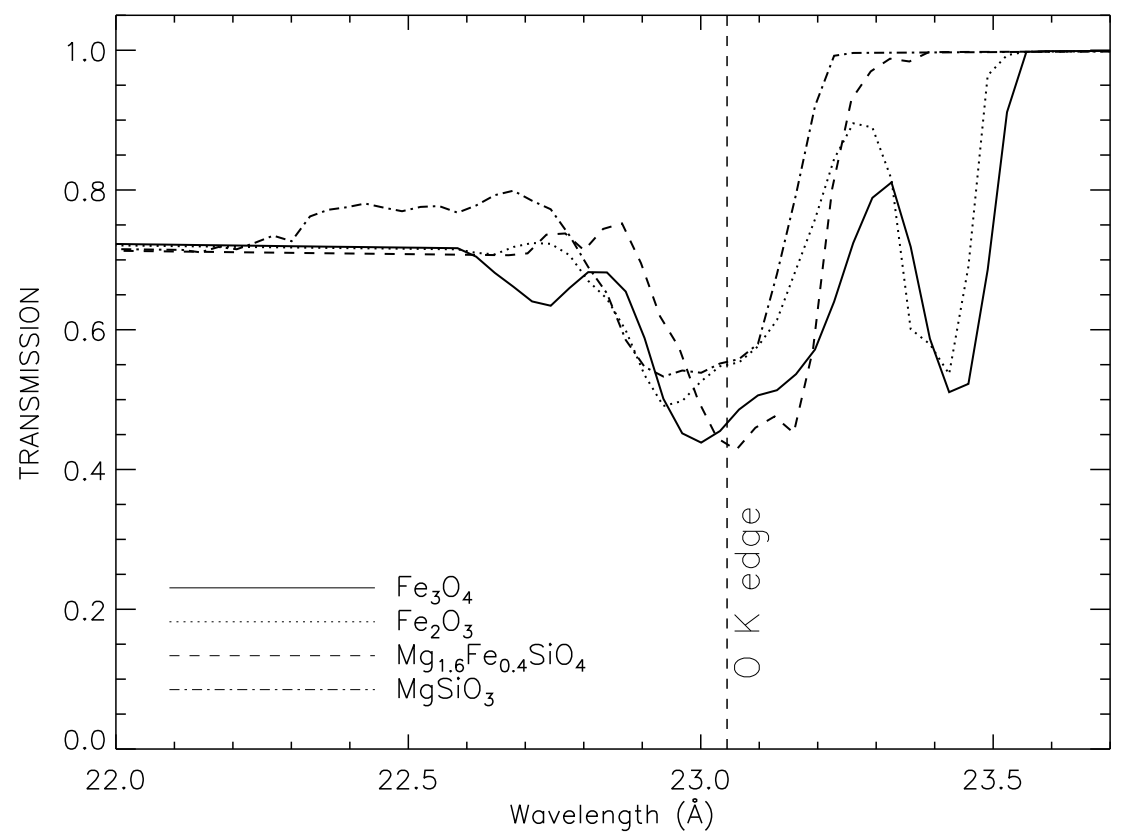

Figure 1: comparison among four different dust compounds in terms of transmission around the oxygen edge. Edges' profiles are taken from the literature (see [13,3], and references therein). The vertical line marks the position of the atomic oxygen edge.

of a bright X-ray source in the Galactic plane, may show in fact a more important contribution of Fe-bearing silicates (Zeegers et al. in prep.).

\section{Laboratory measurements}

It is clear that a solid parametrization of ID in the X-ray must be built on a complete set of measurements for many compounds. In the last two years we have taken action to carry out the necessary measurements. We took care of measuring every edge that a compound would produce in absorption (e.g. $\mathrm{O}, \mathrm{Fe}, \mathrm{Si}$ and $\mathrm{Mg}$ for classical silicates), using the most modern facilities in Europe. We focused also on amorphous silicates, as crystals (commonly measured in laboratories) are in fact no more than 5\% in the diffuse ISM [9]. Amorphous grains, which are the result of reprocessing in the ISM, reflect the true composition of the ID. This pilot set of measurements has been successfully carried out. The first results on the Mg and Si edges, measured using the LUCIA beamline at the Soleil synchrotron, are showed by S. Zeegers (these proceeedings).

\section{The X-ray future of dust}

The current X-ray instruments have optimized performances, in terms of effective area and energy resolution $(E / \Delta E=400-1000)$, in the low-energy $\mathrm{X}$-ray band $(\sim 0.5-2 \mathrm{keV})$. This allows us to study in detail the $\mathrm{K}$ edge of oxygen $(E=0.543 \mathrm{keV})$ and the L edges of iron $(E=0.7 \mathrm{keV})$. These are representative however of a diffuse ID environment. 


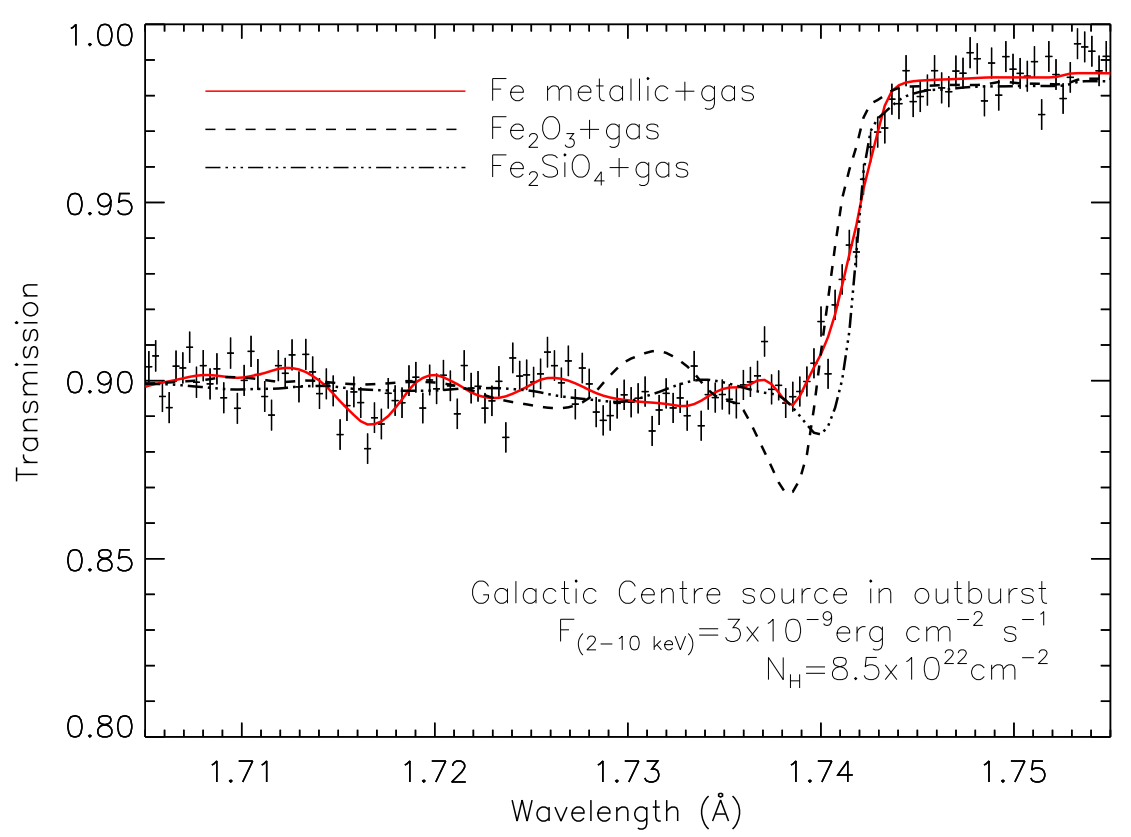

Figure 2: Simulation of Athena + X-ray Integral Field Unit (XIFU) of the hard X-ray spectrum of a highlyabsorbed source in outburst. The high energy resolution (foreseen to be $\sim 2400$ at $6 \mathrm{keV}$ ) will reveal the iron depletion and its inclusion in dust particles. The iron $\mathrm{K}$ edge is at $E=7.1 \mathrm{keV}(1.74 \AA)$. Adapted from [4].

Future X-ray missions (namely Astro-H and Athena+ ) will strongly enhance the sensitivity at harder X-rays $(E>1 \mathrm{keV})$. In particular the $\mathrm{Si} \mathrm{K}(E=1.84 \mathrm{keV})$ and the $\mathrm{Fe} \mathrm{K}(E=7.1 \mathrm{keV})$ edges will be revealed with unprecedented detail in dense Galactic environments $\left(N_{\mathrm{H}}>5 \times 10^{22} \mathrm{~cm}^{-2}\right)$. Given the high extinction of the regions near the Galactic Center (up to $N_{\mathrm{H}} \sim 10^{23} \mathrm{~cm}^{-2}$ ), the study of e.g. iron depletion and its inclusion in dust will be only possible investigating the hard X-ray band (Fig. 2, [4]).

\section{References}

[1] Bradley, J. 2010, LNP, 815, 259

[2] Costantini, E., Freyberg, M. J., \& Predehl, P. 2005, A\&A , 444, 187, C05

[3] Costantini, E., Pinto, C., Kaastra, J. S., et al. 2012, A\&A , 539, A32, C12

[4] Decourchelle, A., Costantini, E., Badenes, C., et al. 2013, arXiv:1306.2335

[5] Draine, B. T. 2003, ApJ, 598, 1026

[6] Draine, B. T. 2009, ASPC, 414, 453

[7] Gail, H.-P. 2010, LNP, 815, 61

[8] Jenkins, E. B. 2009, ApJ , 700, 1299

[9] Kemper, F. et al. 2001, A\&A , 369, 132

[10] Lee, J.C., Xiang, J., Ravel, B., Kortright, J., \& Flanagan, K. 2009, ApJ , 702, 970 
[11] Li, A., \& Draine, B. T. 2001, ApJ , 550, L213

[12] Pedicelli, S., Bono, G., Lemasle, B., et al. 2009, A\&A, 504, 81

[13] Pinto, C., Kaastra, J. S., Costantini, E., \& Verbunt, F. 2010, A\&A , 521, A79

[14] Pinto, C., Kaastra, J.S., Costantini, E., \& de Vries, C. 2013, A\&A, 551, 25

[15] Smith, R. K., Dame, T. M., Costantini, E., \& Predehl, P. 2006, ApJ , 648, 452

[16] Tiengo, A., Vianello, G., Esposito, P., et al. 2010, ApJ , 710, 227

[17] Valencic,L. \& Smith R.K. 2013, ApJ, 1306, 2307

[18] Yao, Y., Zhang, S. N., Zhang, X., \& Feng, Y. 2003, ApJL, 594, L43 